\title{
Predictors of Hospital Mortality in Patients with Acute Coronary Syndrome Complicated by Cardiogenic Shock
}

\author{
Gábor Tamás Szabó ${ }^{1, *}{ }^{\mathbb{D}}$, András Ágoston ${ }^{2}$, Gábor Csató ${ }^{3}$, Ildikó Rácz ${ }^{1}$, Tamás Bárány ${ }^{1}{ }^{1}$, Gábor Uzonyi ${ }^{4}$, \\ Miklós Szokol ${ }^{1}$, Balázs Sármán ${ }^{4}$, Éva Jebelovszki ${ }^{5}$, István Ferenc Édes ${ }^{6}$, Dániel Czuriga ${ }^{1}{ }^{1}$, Rudolf Kolozsvári ${ }^{1}$, \\ Zoltán Csanádi ${ }^{1}$, István Édes ${ }^{1}$ and Zsolt Köszzegi ${ }^{1,2}$ (B)
}

1 Department of Cardiology, Faculty of Medicine, University of Debrecen, 4032 Debrecen, Hungary; iracz73@gmail.com (I.R.); drbaranytamas@gmail.com (T.B.); miklos.szokol@gmail.com (M.S.); dczuriga@med.unideb.hu (D.C.); rudlinghealth@yahoo.com (R.K.); csanadi.zoltan@med.unideb.hu (Z.C.); edes@med.unideb.hu (I.É.); koszegi@med.unideb.hu (Z.K.)

2 The III: Department of Internal Medicine, Szabolcs-Szatmár-Bereg County Hospitals and University Teaching Hospital, 4400 Nyíregyháza, Hungary; agostonandras48@gmail.com

3 Hungarian National Ambulance Service, 1024 Budapest, Hungary; csatogabor@yahoo.com

4 Department of Cardiology, Uzsoki Hospital, 1145 Budapest, Hungary; uzonyi.gabor@uzsoki.hu (G.U.); sarman.balazs@uzsoki.hu (B.S.)

5 Department of Cardiology, Faculty of Medicine, University of Szeged, 6725 Szeged, Hungary; jebelovszki@freemail.hu

6 Heart and Vascular Center, Semmelweis University, 1122 Budapest, Hungary; edes.istvan@kardio.sote.hu

* Correspondence: nszgt@med.unideb.hu

Citation: Szabó, G.T.; Ágoston, A.; Csató, G.; Rácz, I.; Bárány, T.; Uzonyi, G.; Szokol, M.; Sármán, B.; Jebelovszki, É.; Édes, I.F.; et al. Predictors of Hospital Mortality in Patients with Acute Coronary Syndrome Complicated by Cardiogenic Shock. Sensors 2021, 21, 969. https://doi.org/10.3390/ s21030969

Academic Editors: Jasper J. Brugts, Dominic A.M.J. Theuns, Sumant P. Radhoe and Jesse F. Veenis Received: 22 December 2020 Accepted: 26 January 2021 Published: 1 February 2021

Publisher's Note: MDPI stays neutral with regard to jurisdictional claims in published maps and institutional affiliations.

Copyright: () 2021 by the authors. Licensee MDPI, Basel, Switzerland. This article is an open access article distributed under the terms and conditions of the Creative Commons Attribution (CC BY) license (https:// creativecommons.org/licenses/by/ $4.0 /)$.
Abstract: As demonstrated by earlier studies, pre-hospital triage with trans-telephonic electrocardiogram (TTECG) and direct referral for catheter therapy shows great value in the management of out-of-hospital chest pain emergencies. It does not only improve in-hospital mortality in ST-segment elevation myocardial infarction, but it has also been identified as an independent predictor of higher in-hospital survival rate. Since TTECG-facilitated triage shortens both transport time and percutaneous coronary intervention (PCI)-related procedural time intervals, it was hypothesized that even high-risk patients with acute coronary syndrome (ACS) and cardiogenic shock (CS) might also benefit from TTECG-based triage. Here, we decided to examine our database for new triage- and left ventricular (LV) function-related parameters that can influence in-hospital mortality in ACS complicated by CS. ACS patients were divided into two groups, namely, (1) hospital death patients $(n=77)$, and (2) hospital survivors (control, $n=210$ ). Interestingly, TTECG-based consultation and triage of CS and ACS patients were confirmed as significant independent predictors of lower hospital mortality risk (odds ratio (OR) 0.40, confidence interval (CI) 0.21-0.76, $p=0.0049$ ). Regarding LV function and blood chemistry, a good myocardial reperfusion after PCI (high area at risk (AAR) blush score/AAR LV segment number; OR 0.85, CI 0.78-0.98, $p=0.0178$ ) and high glomerular filtration rate (GFR) value at the time of hospital admission (OR 0.97, CI 0.96-0.99, $p=0.0042$ ) were the most crucial independent predictors of a decreased risk of in-hospital mortality in this model. At the same time, a prolonged time interval between symptom onset and hospital admission, successful resuscitation, and higher peak creatine kinase activity were the most important independent predictors for an increased risk of in-hospital mortality. In ACS patients with CS, (1) an early TTECG-based teleconsultation and triage, as well as (2) good myocardial perfusion after PCI and a high GFR value at the time of hospital admission, appear as major independent predictors of a lower in-hospital mortality rate.

Keywords: telemedicine; prehospital triage; acute heart failure; myocardial perfusion

\section{Introduction}

Ischaemic heart disease is one of the common causes of death worldwide and its frequency is increasing [1]. Recent guidelines [2,3] state that the timely diagnosis of acute coronary syndrome (ACS) and the elimination of any delay are the key factors 
of an improved outcome. In most ACS cases, the first medical contact takes place far from hospitals, usually initiated by the paramedic staff. Therefore, in the vast majority of cases, the primary, pre-hospital diagnosis of ACS is based on the typical signs and symptoms of the disease and especially on the electrocardiogram (ECG) analysis [4]. Besides the traditional 12-lead ECG, trans-telephonic ECG (TTECG) can also be applied by the paramedic specialist [2]. In previous studies, ourselves and others have shown that the utilization of the TTECG and TTECG-facilitated triage of patients significantly shortened the intervention time and improved in-hospital mortality in patients with ST-segment elevation myocardial infarction (STEMI) [5,6]. ACS, including STEMI, is one of the main precipitating factors of cardiogenic shock (CS) [7,8]. It has been estimated that $5-15 \%$ of patients with ACS experience signs and symptoms of CS $[9,10]$. Although the prognosis of CS has gradually improved over the past few years, the hospital mortality rate is still high $(20-40 \%)$, despite advances in early revascularization and the implementation of new drugs and mechanical circulatory support devices [10]. Some factors that affect mortality in ACS patients with CS have already been established. Among others, these are advanced age, anoxic brain damage, low left-ventricular ejection fraction (LVEF), deterioration in renal function, and resuscitation $[7,8,10]$. A patient displaying ACS and CS should be triaged to a fast track for early revascularization, with the aim of maintaining perfusion to prevent organ dysfunction.

Since the TTECG-facilitated triage of STEMI patients has shortened both transport time and percutaneous coronary intervention (PCI) procedural time intervals, and, at the same time, improved hospital survival [6], we have decided to examine our ACS patient database for CS to look for factors that affect mortality. In particular, our aim was to find new triage- and LV function-related independent predictors that may influence in-hospital mortality in CS complicating ACS.

In order to put our hypothesis to the test, we set out to retrospectively evaluate our database of ACS patients with CS $(n=287)$ for: (1) TTECG procedures, (2) various LV functional data and (3) demographic factors, comorbidities, and cardiac risk factors. Our main objective was to examine the difference between the hospital death group and the hospital survival group with the expectation that the early TTECG-facilitated triage and improved pre-hospital therapy of patients would lead to better perfusion, LV function, and improved patient survival.

\section{Materials and Methods}

\subsection{Data Collection}

The study was conducted between 1st January 2009 and 31st December 2012 at the Northern Great Plain region of Hungary as a collaborative effort between the Department of Cardiology, University of Debrecen, Hungary and the Hungarian National Ambulance Service. During this time, 2506 patients were transferred to the regional PCI centre (Department of Cardiology, University of Debrecen, Debrecen, Hungary) with a diagnosis of ACS. The patient flow is depicted in Figure 1.

Upon arrival at the PCI centre, each patient was promptly interviewed and examined by a cardiologist and the diagnosis of ACS was established. At the time of admission, laboratory blood tests were run via a series of creatine kinase (CK) measurements, renal and liver functions, as well as blood count. Based on serial CK measurements, the peak $\mathrm{CK}\left(\mathrm{CK}_{\max }\right)$ value was determined. Following bedside echocardiography, patients were transferred to the catheterization laboratory for coronary angiography, and, when necessary, for PCI.

Among ACS patients, altogether 287 CS cases were registered (Killip class IV) [11]. The diagnosis of CS was based on hypotension (systolic blood pressure $<90 \mathrm{mmHg}$ ) with signs of hypoperfusion. For all ACS patients with CS, immediate echocardiography, coronary angiography, and, if possible, coronary revascularization were carried out. After fluid challenge, all ACS patients with CS received vasopressor therapy and an inotropic agent, when necessary. In the case of respiratory failure (hypoxemia or hypercapnia), the 
patients were intubated and ventilated. Since the study was carried out between 2009 and 2012, most of the patients (282 patients) also received device therapy in the form of intra-aortic balloon pump (IABP) support. Recently, it has been shown in the IABP-SHOCK II trial that the use of an IABP did not actually improve outcomes in patients suffering from ACS and CS [12,13]. Therefore, in recent guidelines the routine use of an IABP is not recommended $[10,14]$.

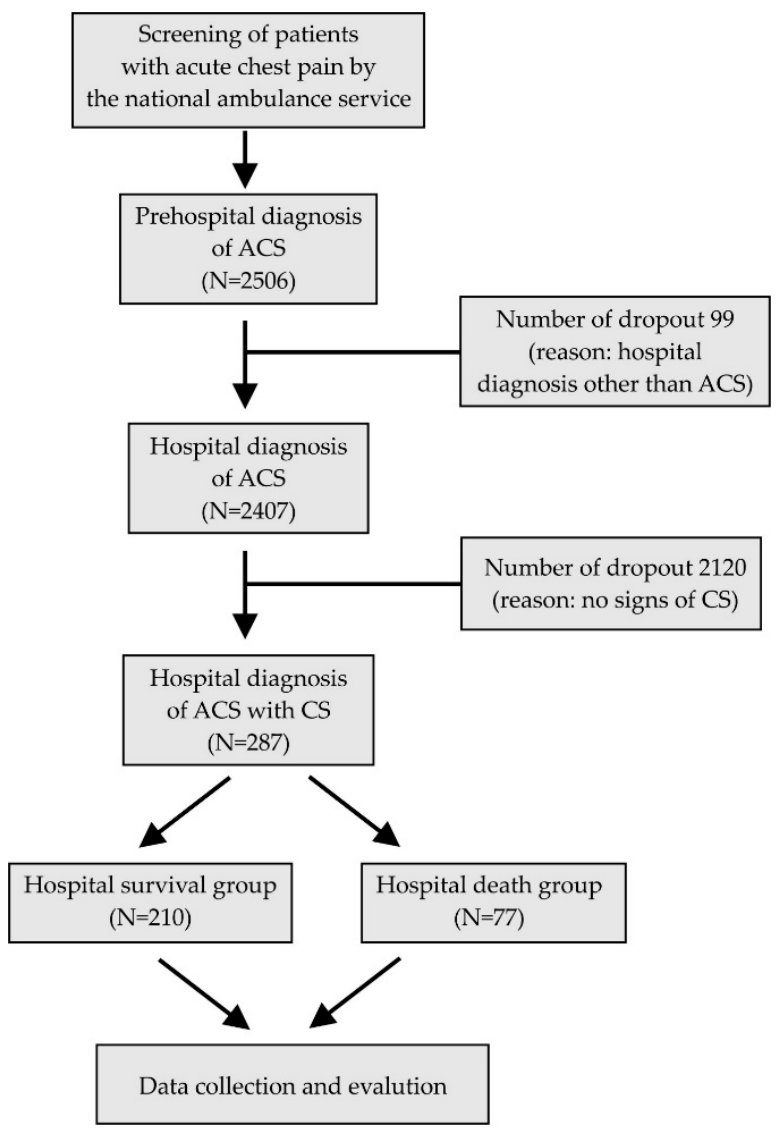

Figure 1. CONSORT diagram showing patient flow at each stage of the data collection. ACS = acute coronary syndrome; $\mathrm{CS}=$ cardiogenic shock.

ACS patients with CS were assigned to the following two groups: (1) hospital death patients $(n=77)$ and (2) hospital survivors (control, $n=210)$. The primary aim of this study was to examine the efficacy outcomes of triage- and LV function-related parameters affecting in-hospital mortality.

The TTECG methodology was previously described in detail [6]. TTECG was performed with a portable, 12-lead, battery-operated system (HeartView P12/8 Plus by Aerotel). The device was supplemented with 3 external, cable-connected electrodes and $4 \mathrm{em}$ bedded electrodes on the back of the main unit. This layout allowed the recording of both limb and precordial leads. A $2.5 \mathrm{~s}$ interval of each lead and a $10 \mathrm{~s}$ interval of the rhythm strip (lead II) were recorded with a sampling rate of 375 samples/s, producing a standard 12-lead ECG layout. The radiotelephone system of the Hungarian National Ambulance Service (Tetra) was used for data transmission.

Paramedic specialists participating in the trial were trained for emergency cardiac service and advanced cardiovascular life support. Before the study, the specialists were instructed how to evaluate patients with chest pain (with a presumptive diagnosis of ACS) at the scene and administer acetylsalicylic acid, sodium heparin, narcotics, and other medications if necessary. All units were uniformly equipped with both conventional ECG and portable TTECG systems. The conventional ECG machine recorded 12 leads in 4 consecutive steps at a standard paper speed $(25 \mathrm{~mm} / \mathrm{s})$. Conventional ECG was 
always recorded by the emergency team at the first contact. However, during the period of the study, it was not obligatory, just an option for the paramedic team to record and transmit a TTECG to the PCI centre. Some teams obtained and transferred TTECGs from all ACS patients, while other teams did so only if they encountered problems with the clinical diagnosis, medical treatment, and the interpretation of the ECG. Typically, when the patient was located farther away from the PCI centre (rural areas), TTECG-based triage was used more frequently by emergency teams. Once the TTECG signal was transmitted to the PCI centre, the patient's relevant clinical data were discussed via teleconsultation.

Coronary angiography investigations were performed with the help of a Philips Integris CV device, cine loops were recorded at 15 frames/s using $300 \mathrm{mg}$ iodine/mL non-ionic contrast material. The primary $\mathrm{PCI}$ procedure was performed in a standard way. The left ventricular (LV) area at risk (AAR) calculation was based on the analysis of coronary angiograms from multiple projections with a validated computer software (Holistic Coronary Care; HCC) [15]. To represent most of the individual variations of the coronary circulation, this program uses a modified coronary classification method, resulting in 12 different coronary artery circulation types.

As the blood supply of the myocardium can be more adequately quantified by evaluating myocardial perfusion than visualizing the epicardial flow on the angiogram [16], myocardial reperfusion after PCI was assessed by the myocardial blush grade obtained using a computer program (Quantitative Blush Evaluator; QuBE) [17,18]. The blush grade results (QuBE score) were expressed in arbitrary units for the whole LV (LV QuBE score) or for segments in the AAR (total AAR QuBE score and AAR QuBE score/AAR segments number).

All patients underwent an echocardiographic examination before and after the intervention, using an Acuson-Sequoia device with a $3.5 \mathrm{MHz}$ harmonic imaging transducer. LV ejection fraction (LVEF) calculation was performed using the Simpson's formula of the software package.

The time interval between symptom onset and hospital admission was the duration of time between the onset of typical chest pain and the arrival of the ambulance service unit at the PCI centre. Door-to-balloon time was defined as the time interval between the arrival of the ambulance service unit at the PCI centre and the inflation of the intracoronary balloon in the catheterization laboratory.

The study was conducted in accordance with the Declaration of Helsinki. Study data were collected after receiving the written consent of the patients or their relatives. Data management and collection procedures were approved by the institutional review boards of the Department of Cardiology, University of Debrecen, Hungary and the Hungarian National Ambulance Service.

\subsection{Statistical Analysis}

A statistical analysis was performed by the GB-STAT v8.0 program. Depending on the type of variable in question, various statistical approaches were applied: calculation of the mean and standard deviations (SD) or calculation of the absolute and relative frequencies. Normally distributed continuous variables were compared by using Student's $t$ test at an $\alpha$-level of $5 \%$; while comparative analysis of categorical variables was carried out using nonparametric statistics (Wilcoxon-Mann-Whitney test) at a $\alpha$-level of $5 \%$.

All predetermined variables between the hospital death group and the hospital survival group were assessed by applying a univariate logistic regression model. Odds ratios and $95 \%$ confidence intervals (CI) were calculated. Variables characterized by a $p<0.20$ in the univariate analysis were selected for multiple regression and were quantified for adjusted odds ratios and CIs for in-hospital mortality. A $p$ value of $<0.05$ was regarded as significant. For cumulative survival analysis, the Cox regression model was used. 


\section{Results}

Altogether, 287 ACS patients with CS were involved in the study (77 patients in the hospital death group and 210 patients in the hospital survival group). The total in-hospital mortality was $26.83 \%$ (77 patients). The cause of death was progression of CS, asystole, cardiac tamponade, arrhythmias, stent thrombosis, and infection. The frequency of death was highest in the first week of hospitalization (47 deaths occurred in the first week). After day 48, no hospital death occurred in this patient's cohort (Figure 2).

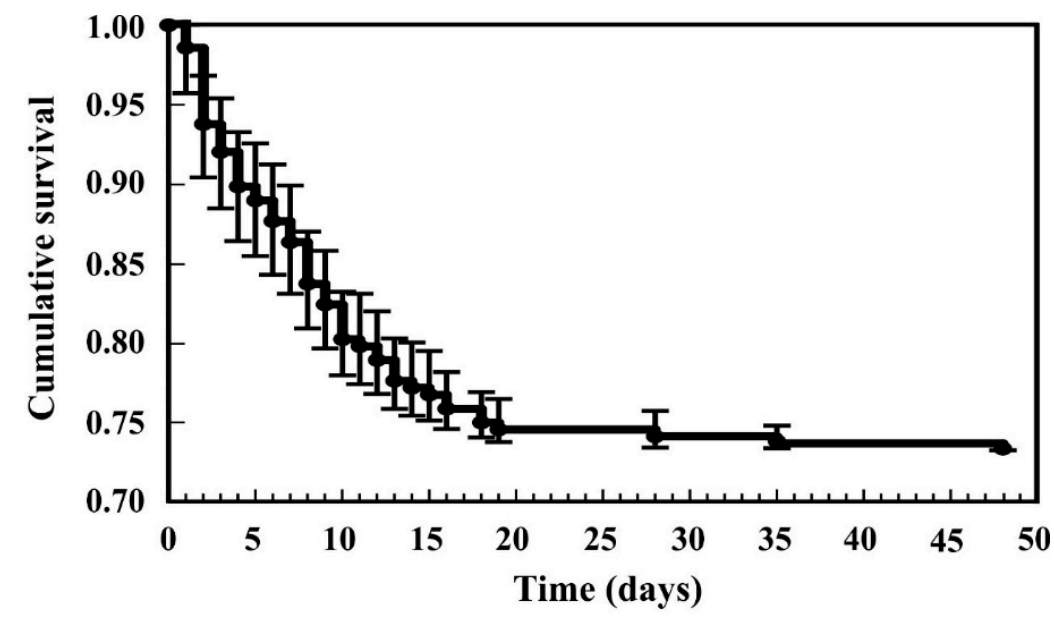

$\begin{array}{lllllllllll}\text { Number at risk } & 287 & 235 & 159 & 80 & 37 & 21 & 9 & 4 & 2 & 2\end{array}$

Figure 2. The Kaplan-Meier curve depicts the in-hospital survival rate (value $\pm 95 \%$ confidence intervals) of all the acute coronary syndrome patients with cardiogenic shock in the first 48 days.

The one-year mortality of ACS patients with CS was 33.45\% (96 patients). The mean time spent in hospital was $11.45 \pm 7.86$ days, with an average duration of IABP treatment of $4.82 \pm 3.82$ days. The number of IABP-related vascular complications was 16 cases (pseudoaneurysm, leg ischaemia, thromboembolic complications, or severe haematoma).

Table 1 shows the baseline characteristics of the hospital death and survival groups.

The two groups did not appear to be significantly different with regard to risk factors and earlier medical history. Still, patients in the hospital death group were moderately, but not significantly $(p=0.1425)$ older than the hospital survivors. An examination of the blood chemistry of patients revealed significantly higher $\mathrm{CK}_{\max }$ and a lower baseline glomerular filtration rate (GFR) values in the hospital death group compared to the survivor group $(p=0.0038$ and $p<0.0001$, respectively). Significantly more $(p=0.0104)$ TTECGbased consultations were performed in the hospital survival group than in the hospital death group. As expected, the rates of successful pre-hospital and hospital resuscitations were slightly higher in the hospital death group in comparison with the survivor group, nevertheless, the difference did not prove to be statistically significant. The examination of time intervals between symptom onset and hospital admission (Table 1 ) showed significant variations; however, this parameter was significantly longer $(p<0.0001)$ in the hospital death group compared to survivors. The selective analysis of hospital death group data revealed that TTECG was associated with significantly $(p<0.0065)$ shortened time intervals (in hours) between symptom onset and hospital admission (mean \pm SD: hospital death with TTECG $6.75 \pm 6.77 \mathrm{~h}, n=21$; hospital death without TTECG $34.98 \pm 62.49 \mathrm{~h}, n=56$; all hospital death $26.63 \pm 54.93 \mathrm{~h}, n=77)$. However, this phenomenon was substantially mitigated in this group due to the low number of patients with TTECG.

The effect of TTECG-based triage on the time interval between symptom onset and hospital admission was also directly examined. For this purpose, we created two groups: TTECG group $(n=116)$ and control group without TTECG $(n=171)$. The following results (mean \pm SD, in hours) were noted: TTECG group, $9.44 \pm 11.21$; control group, $23.77 \pm 48.63$ 
$(p<0.0001)$. Based on these data, it is obvious that TTECG-mediated triage was associated with a drastically shortened time interval between symptom onset and hospital admission.

Table 1. Clinical characteristics of acute coronary syndrome patients with acute heart failure.

\begin{tabular}{|c|c|c|c|}
\hline & Hospital Death Group $(n=77)$ & Hospital Survival Group $(n=210)$ & $p$ Value \\
\hline \multicolumn{4}{|l|}{ General } \\
\hline Age (yrs) & $66.04 \pm 12.56$ & $63.60 \pm 11.98$ & 0.1425 \\
\hline Men $(\%)$ & 59.74 & 61.90 & 0.7318 \\
\hline TTECG-based consultation (\%) & 27.27 & 45.71 & 0.0104 \\
\hline $\begin{array}{l}\text { Time interval between symptom onset } \\
\text { and hospital admission (h) }\end{array}$ & $26.63 \pm 54.93$ & $13.16 \pm 25.35$ & $<0.0001$ \\
\hline 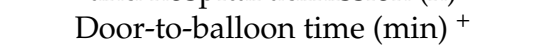 & $68.24 \pm 39.03$ & $60.06 \pm 29.25$ & 0.1369 \\
\hline Resuscitation $(\%)$ & 24.67 & 14.28 & 0.1775 \\
\hline $\operatorname{LVEF}(\%)$ & $30.28 \pm 8.37$ & $36.96 \pm 8.67$ & $<0.0001$ \\
\hline AAR $(\%)$ & $66.62 \pm 24.59$ & $61.95 \pm 24.67$ & 0.1571 \\
\hline LV QuBE score (arbitrary units) $§$ & $115.50 \pm 33.31$ & $160.13 \pm 38.70$ & $<0.0001$ \\
\hline AAR QuBE score (arbitrary units) $\S$ & $55.95 \pm 30.16$ & $90.11 \pm 46.08$ & $<0.0001$ \\
\hline $\begin{array}{c}\text { AAR QuBE score/AAR segment } \\
\text { number (arbitrary units) } \S\end{array}$ & $4.97 \pm 2.28$ & $8.89 \pm 3.43$ & $<0.0001$ \\
\hline \multicolumn{4}{|l|}{ Blood Chemistry } \\
\hline GFR $(\mathrm{mL} / \mathrm{min})$ & $52.71 \pm 23.39$ & $66.25 \pm 22.33$ & $<0.0001$ \\
\hline $\mathrm{CK}_{\max }(\mathrm{U} / \mathrm{L})$ & $5141.27 \pm 8247.84$ & $2166.47 \pm 2607.36$ & 0.0038 \\
\hline Haemoglobin (g/L) & $130.17 \pm 21.18$ & $135.56 \pm 19.82$ & 0.0625 \\
\hline Thrombocyte number $\times 10^{3}(\mu \mathrm{L})$ & $241.50 \pm 72.90$ & $259.10 \pm 72.70$ & 0.2459 \\
\hline \multicolumn{4}{|l|}{ Previous Cardiac Risk Factors (\%) } \\
\hline Smoking & 29.87 & 31.42 & 0.8178 \\
\hline Hypertension & 64.93 & 68.09 & 0.6052 \\
\hline Diabetes mellitus & 35.06 & 32.38 & 0.7616 \\
\hline PAD & 19.48 & 25.71 & 0.3931 \\
\hline \multicolumn{4}{|l|}{$\begin{array}{c}\text { Proportion of Patients (\%) with a } \\
\text { Previous History of }\end{array}$} \\
\hline Myocardial infarction & 23.37 & 28.57 & 0.5002 \\
\hline Congestive heart failure & 16.88 & 20.00 & 0.7318 \\
\hline PCI & 10.38 & 9.52 & 0.9105 \\
\hline Coronary artery bypass graft surgery & 3.89 & 3.33 & 0.7961 \\
\hline
\end{tabular}

Values are means $\pm \mathrm{SD}$ or percentages of subjects. AAR $=$ area at risk; GFR $=$ glomerular filtration rate; $\mathrm{LV}=$ left ventricle; $\mathrm{LVEF}=$ left ventricular ejection fraction; $\mathrm{PAD}=$ peripheral artery disease; $\mathrm{PCI}=$ percutaneous coronary intervention; $\mathrm{QuBE}=$ myocardial blush grade; TTECG $=$ transtelephonic ECG. In this instance, the $p$ value refers to differences between the hospital death group and the hospital survival group. ${ }^{+}$The door to balloon time was available only if the PCI has been performed ( $n=64$ and 155 in the hospital death group and hospital survival group, respectively). ${ }^{\S}$ In case of QuBE calculations the number of patients $(n)$ were 66 and 156 in the hospital death group and hospital survival group, respectively.

Stent procedural data and the medical therapy introduced in the acute phase of the disease are summarized in Table 2.

In the case of 214 patients, the clinical diagnosis was CS and STEMI. The remaining cases (73 patients) were classified as CS and non-ST-elevation myocardial infarction (NSTEMI). Stents were deployed in $76.31 \%$ of the patients (hospital death group: $83.12 \%$, survivor group: $73.81 \%$ ). The door-to-balloon time of PCI patients was slightly but not significantly $(p=0.1369)$ longer in the hospital death group, compared to hospital survivors. Since the study was carried out between 2009 and 2012, a relatively low percent of drugeluting stents was used (hospital death group: $5.19 \%$, survivor group: $10.00 \%$ ). In the case of 68 patients, no stent implantation was carried out. The reasons for not implanting stents were the following: 48 patients were referred for immediate cardiac surgery (three-vessel coronary artery disease and mechanical complications), 17 patients received balloon- or drug-eluting balloon angioplasty, 13 patients had multivessel disease with chronic total occlusions (not suitable for revascularization) and in 10 patients the stent implantation was technically not successful. Some patients had more than one reason for not having stent 
implantation. Considering stent procedural details, no significant difference was found between the two groups, as demonstrated in Table 2.

Table 2. Stent procedural details and medical therapy in the acute phase (first week).

\begin{tabular}{|c|c|c|c|}
\hline & $\begin{array}{l}\text { Hospital Death } \\
\text { Group }(n=77)\end{array}$ & $\begin{array}{l}\text { Hospital Survival } \\
\text { Group }(n=210)\end{array}$ & $p$ Value \\
\hline \multicolumn{4}{|l|}{ Stent procedural details } \\
\hline Proportion of STEMI patients (\%) & 83.12 & 71.43 & 0.1293 \\
\hline One-vessel disease (\%) & 64.93 & 54.29 & 0.1069 \\
\hline Proportion of stented patients (\%) & 83.12 & 73.81 & 0.1010 \\
\hline Stent/patient (mean $\pm \mathrm{SD})$ & $1.24 \pm 0.50$ & $1.28 \pm 0.53$ & 0.2945 \\
\hline Drug-eluting stent (\%) & 5.19 & 10.00 & 0.1010 \\
\hline${ }^{*} \mathrm{LM}(\%)$ & 3.13 & 3.23 & 0.9693 \\
\hline${ }^{*} \operatorname{LAD}(\%)$ & 68.75 & 76.77 & 0.2168 \\
\hline${ }^{*} \mathrm{CX}(\%)$ & 18.75 & 23.87 & 0.4093 \\
\hline *RCA (\%) & 29.68 & 21.29 & 0.1851 \\
\hline \multicolumn{4}{|l|}{$\begin{array}{l}\text { Medical Therapy in the } \\
\text { Acute Phase }(\%)\end{array}$} \\
\hline Inotropes (dobutamine) & 97.40 & 94.76 & 0.7318 \\
\hline $\begin{array}{c}\text { Vasopressor } \\
\text { (norepinephrine/dopamine) }\end{array}$ & 100.00 & 100.00 & 1.0000 \\
\hline Levosimendan & 9.19 & 10.00 & 0.9597 \\
\hline GP IIb/IIIa inhibitor & 51.95 & 44.76 & 0.2708 \\
\hline
\end{tabular}

All patients received vasopressors and/or inotropes (there was no significant difference between the two groups). Moreover, no significant difference was observed when comparing the hospital death group with the survival group from the aspect of levosimendan and glycoprotein (GP) IIb/IIIa inhibitor (eptifibatide) administration during the acute phase of the disease (first week).

Examining the coronary angiograms of patients using the HCC program revealed that the AAR of the LV was slightly, but not significantly larger in the hospital death group than in the survivor group ( $p=0.1571$, Table 1$)$. The above finding was in line with the significantly higher $\mathrm{CK}_{\max }$ and lower LVEF values found by echocardiography in the hospital death group.

In the hospital survivor group, significantly higher QuBE scores values (LV QuBE score, total AAR QuBE score and AAR QuBE score/AAR segments number) were noted following PCI (Table 1) than in the hospital death group. The significant differences in QuBE scores between the two groups suggest better myocardial reperfusion and microvascular function in the survivor group.

All the predetermined parameters between the two groups were evaluated by the univariate log-rank test (Figure 3), also, odds ratios and CIs for in-hospital mortality rate were calculated. Applying the univariate statistical method, several blood chemistry parameters, LV functional data, time interval between symptom onset and hospital admission, resuscitation, and the TTECG-based consultation turned out to exert significant effect on the in-hospital mortality rate of ACS patients with CS. 
TTECG based consultation $(p=0.0021)$

Time to symptom onset to hospital $(p<0.0001)$

Door to balloon time $(p=0.1024)$

Age $(p=0.1276)$

Male gender $(p=0.6841)$

$\operatorname{AAR}(\mathbf{p}=\mathbf{0 . 1 3 3 3})$

AAR QuBE $(\mathbf{p}<0.0001)$

\section{Decreased risk Increased risk}

AAR QuBE/AAR segment $(\mathbf{p}<0.0001)$

LV QuBE (p<0.0001)

LVEF $(p<0.0001)$

Resuscitation $(\mathrm{p}=\mathbf{0 . 0 4 4 3})$

Smoking $(p=0.7687)$

Hypertension $(p=0.5281)$

Diabetes $(p=0.7077)$

$\operatorname{PDA}(p=0.2322)$

$\mathrm{CK}_{\max }(\mathrm{p}=\mathbf{0 . 0 0 0 6})$

Hemoglobin $(\mathrm{p}=\mathbf{0 . 1 1 3 1})$

GFR $(\mathbf{p}<0.0001)$

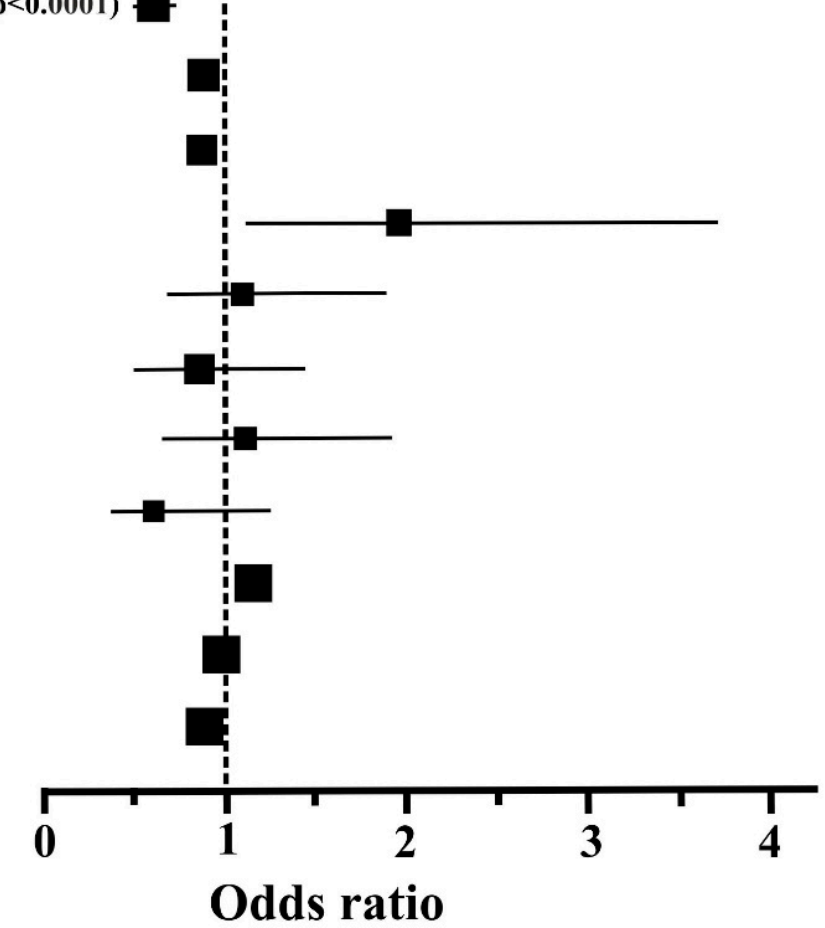

Figure 3. Odds ratios and $95 \%$ confidence intervals for hospital mortality in the individual subgroups, defined on the basis of baseline characteristics, blood chemistry, and percutaneous coronary intervention-related procedural data. Only selected parameters, and variables displaying a $p$ value of $p<0.2$ with the comparative analysis are shown. The sizes of the symbols reflect the number of patients in each group. For some parameters (time to symptom onset to hospital, door to balloon time, age, AAR, AAR QuBE, LV QuBE, LVEF, $\mathrm{CK}_{\max }$, hemoglobin, and GFR), confidence intervals are within the symbols. $\mathrm{AAR}=$ area at risk; $\mathrm{CK}_{\max }=$ peak creatine kinase level; $\mathrm{GFR}=$ glomerular filtration rate; $\mathrm{LV}=$ left ventricle; $\mathrm{LVEF}$ = left ventricular ejection fraction; $\mathrm{PAD}$ = peripheral artery disease; $\mathrm{QuBE}=$ blush score; TTECG = transtelephonic electrocardiogram.

Our data analysis showed that prolonged time interval between symptom onset and hospital admission, successful resuscitation and higher $\mathrm{CK}_{\max }$ activity were linked to significantly increased odds ratios for mortality (Figure 3). Additionally, higher LVEF values, good reperfusion after PCI (high QuBE scores), and TTECG-based triage significantly lowered the odds ratios of in-hospital mortality (Figure 3).

Variables characterized by a $p<0.20$ in the univariate analysis were selected for multiple regression and were quantified for adjusted odds ratios and CIs for in-hospital mortality. Interestingly enough, the TTECG-based consultation and triage were confirmed 
as significant independent predictors of lower risk (odds ratio 0.40 , CI 0.21-0.76, $p=0.0049$ ). Regarding LV function and blood chemistry, a high AAR QuBE score/AAR segment number ratio (odds ratio 0.85, CI 0.78-0.98, $p=0.0178$ ) and higher GRF values (odds ratio 0.97 , CI $0.96-0.99, p=0.0042$ ) at the time of admission were the most important independent predictors of decreased in-hospital mortality rate in this model. At the same time, prolonged time interval between symptom onset and hospital admission (odds ratio 1.010, CI 1.004-1.014, $p=0.0006$ ), successful resuscitation (odds ratio 1.58, CI 1.01-3.10, $p=0.0411$ ) and high $\mathrm{CK}_{\max }$ values (odds ratio $1.16, \mathrm{CI} 1.04-1.30, p=0.0084$ ) were the major independent predictors of an increased risk of in-hospital mortality rate.

All other parameters which were below $p<0.20$ in the univariate analysis (i.e., LV QuBE score, AAR QuBE score, door-to-balloon time, LVEF, age, and AAR) did not prove to be significant independent predictors of the in-hospital mortality rate in our model.

\section{Discussion}

In previous studies, TTECG-based consultation with cardiologists contributed to bringing about a significantly lower in-hospital mortality rate of STEMI patients $[5,6]$. Moreover, TTECG-based triage has been identified as an independent predictor of decreased hospital mortality [19]. This result was somewhat unexpected, and it was presumed that the benefits related to TTECG-based consultation were associated with more favourable pre-hospital medical therapy, as well as shortened transport and PCI procedural times.

In the present study, we have shown that even in the most severe subgroup of ACS patients (CS and ACS), TTECG-facilitated triage had beneficial effects on the in-hospital survival rate. ECG changes in ACS are known to be highly dynamic. Therefore, the early acquisition and transmission of ECG data in ACS can provide useful and critical information to increase the accuracy of the diagnosis. With reference to recent guidelines, a 12-lead ECG is indispensable, and it must be interpreted as soon as possible at the time of the first medical contact of suspected ACS patients, to facilitate an early diagnosis (class IC recommendation), triage, and therapy [2,3]. To this end, TTECG, combined with teleconsultation, appears to be an optimal tool. It was previously shown that early prehospital triage with telemedicine in subjects with STEMI was linked to higher rates of timely reperfusion [20] and lower mortality rates in both observational studies $[6,21,22]$ and meta-analyses $[23,24]$. These unexpected findings on mortality rates were suggested to be mediated by reduced ischaemic time and better pre-hospital care, resulting from TTECGbased triage [25]. Corresponding to these findings, the present study also confirms that TTECG-based teleconsultation and triage are independent predictors of lower in-hospital mortality rate, and this also applies for high-risk ACS patients with CS.

What could be the causal relationship between TTECG-based triage and improved survival in ACS patients with CS? Based on our current data, TTECG-based triage was associated with a drastically reduced time interval between symptom onset and hospital admission. Evidently, the earlier the reperfusion, the better the myocardial function (i.e., "time is muscle"). Moreover, other important time intervals (door-to-sheath insertion and door-to-balloon time) have also been found to be reduced by TTECG, as shown in both the present study and previous investigations [6]). Finally, yet another important benefit of TTECG-based triage could be that prehospital pharmacotherapy is discussed by the emergency personnel and the PCI centre staff via teleconsultation [6,19].

The hospital medical therapy in our study was administered according to current guidelines [10]. Each patient in the two groups received inotropes and/or vasoconstrictors. After the stabilization of blood pressure $(>90 / 70 \mathrm{mmHg})$, levosimendan was initiated when necessary [26].

It is generally accepted that total occlusions and intracoronary thrombi are directly linked to lower coronary flow, myocardial damage, and loss of function. At the same time, improved coronary flow leads to better myocardial protection and function; consequently, to increased survival rate. Recent ESC Guidelines on myocardial revascularization [14] and acute and chronic heart failure [10] underline the significance of early and complete 
revascularization in order to improve perfusion. The QuBE score values have been shown to have a close correlation with perfusion and microvascular function [18]. Moreover, it was noted that the QuBE score provides a useful surrogate endpoint in trials of therapies that attempt to improve myocardial reperfusion [17]. Indeed, in accordance with the guidelines' proposal, our current results indicate that better perfusion in the affected myocardium (high AAR QuBE score/AAR segment number) after PCI is an important determinant of the survival rate of ACS patients with CS.

Cardiogenic shock is initially characterized by a failure to maintain global oxygen delivery. However, the progression of CS is associated with derangement in the regulation of regional blood flow, microcirculatory abnormalities, and cellular hypoxia [27]. Early restoration of myocardial perfusion may limit the development of microcirculatory and cellular abnormalities. In accordance with this approach, multiple registries [28,29] and clinical studies [30] have confirmed the effectiveness (significant decrease in mortality) of early revascularization in CS. In fact, the SHOCK trial failed to meet the primary endpoint (lowering 30-day mortality) with early revascularization in comparison to initial medical treatment. However, there was a significant mortality reduction after 6 months, 1 year, and 6 years $[31,32]$.

In the present study, the prolonged time interval between symptom onset and hospital admission, the successful resuscitation of ACS patients and high $\mathrm{CK}_{\max }$ value were identified as useful independent predictors for in-hospital mortality rate. These findings correspond to the results demonstrated by previous reports $[7,10]$.

\section{Conclusions}

The main goal of our present study was to determine the impact of various parameters (prehospital TTECG-based teleconsultation, LV function, coronary perfusion, and blood chemistry) on mortality in a retrospective analysis of patients suffering an ACS episode complicated with CS, treated with guideline-based complex therapy. Our research is the first to demonstrate that the TTECG-facilitated diagnosis and triage of patients is an important independent predictor of a higher in-hospital survival rate in this population.

Previously, several studies have shown that the prehospital telemedicine triage of ACS patients speeds up diagnosis while optimizing first-line medical therapy, especially if cardiologists are also involved in the prehospital teleconsultation [5,6]. Furthermore, we have shown that telemedicine facilitates the more efficient organization of patient transport and the preparation of hospital staff for emergent PCI, thereby reducing the time necessary for revascularization. Early coronary artery revascularization results in better LV function and CS outcomes, a finding also supported by the results of our current study. We also showed that improved perfusion of the affected myocardium after PCI is an important determinant of patient survival rates. Moreover, a high GFR value at the time of hospital admission was identified as an independent predictor of better survival, highlighting the importance of preserved function in other organs than the heart in favor of an improved clinical outcome.

To summarize, TTECG-based teleconsultation plays an indisputable role in the establishment of a timely ACS diagnosis and rapid transport of patients; hence, the prompt revascularization of the infarct-related artery and ultimately, the preservation of myocardial function and improved survival of patients with CS. Thus, in the future, it is encouraged to exploit the beneficial effects of telemedicine not only in ambulatory settings, but in the care of acute cardiac events as well.

\section{Limitations}

A potential limitation of this study is that all data analyses were performed on a retrospective basis and on a relatively small sample size (hospital death group; $n=77$ ). It should be emphasized, however, that during data collection, a relatively long inclusion time (4 years) was applied and all ACS patients with CS were included in the database. The respective groups (hospital death group and survival group) were considerably well- 
matched regarding age, gender, risk factors, previous medical history, medical therapy, and stent procedural details.

As the data collection period was 2009-2012, new improvements may have appeared since then both in pharmacological and interventional therapies (new drug-eluting stents or drugs) that were not taken into consideration in our study. Furthermore, other relevant variables may not have been incorporated into our model either.

Author Contributions: Conceptualization, G.T.S., D.C. and Z.K.; methodology, G.T.S., D.C., Z.K. and I.É.; software, G.T.S. and D.C.; validation, Z.C.; formal analysis, Z.K., D.C. and I.É.; investigation, G.T.S., A.Á., Z.K., I.R. and D.C.; resources, G.C., T.B., G.U., M.S., B.S., É.J., I.F.É. and R.K.; data curation, R.K. and G.T.S.; writing—original draft preparation, G.T.S.; writing—review and editing, I.É., D.C. and Z.K.; visualization, D.C. and G.T.S.; supervision, Z.C., Z.K. and I.É.; project administration, I.É.; funding acquisition, I.É. All authors have read and agreed to the published version of the manuscript.

Funding: This publication was supported by the GINOP-2.3.2-15-2016-00043 project. The project was co-financed by the European Union and the European Regional Development Fund.

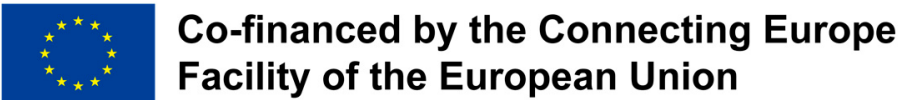

Institutional Review Board Statement: The study was conducted according to the guidelines of the Declaration of Helsinki, and approved by the Institutional Review of University of Debrecen (protocol code MU068, 02.11.2019).

Informed Consent Statement: Informed consent was obtained from all subjects involved in the study.

Data Availability Statement: The data presented in this study are available on request from the corresponding author. The data are not publicly available due to privacy.

Acknowledgments: The authors are indebted to Ilana David from IMS Ltd. for providing the digital database of TTECG and two members Kornélia Kiss and Tamás Ivánfai who assisted in the data collection.

Conflicts of Interest: The authors declare no conflict of interest.

\section{References}

1. Benjamin, E.J.; Muntner, P.; Alonso, A.; Bittencourt, M.S.; Callaway, C.W.; Carson, A.P.; Chamberlain, A.M.; Chang, A.R.; Cheng, S.; Das, S.R.; et al. Heart Disease and Stroke Statistics-2019 Update: A Report From the American Heart Association. Circulation 2019, 139, e56-e528. [CrossRef] [PubMed]

2. Ibanez, B.; James, S.; Agewall, S.; Antunes, M.J.; Bucciarelli-Ducci, C.; Bueno, H.; Caforio, A.L.P.; Crea, F.; Goudevenos, J.A.; Halvorsen, S.; et al. 2017 ESC Guidelines for the management of acute myocardial infarction in patients presenting with STsegment elevation: The Task Force for the management of acute myocardial infarction in patients presenting with ST-segment elevation of the European Society of Cardiology (ESC). Eur. Heart J. 2018, 39, 119-177. [CrossRef] [PubMed]

3. Collet, J.P.; Thiele, H.; Barbato, E.; Barthélémy, O.; Bauersachs, J.; Bhatt, D.L.; Dendale, P.; Dorobantu, M.; Edvardsen, T.; Folliguet, T.; et al. 2020 ESC Guidelines for the management of acute coronary syndromes in patients presenting without persistent ST-segment elevation. Eur. Heart J. 2020. [CrossRef] [PubMed]

4. Chang, A.M.; Fischman, D.L.; Hollander, J.E. Evaluation of Chest Pain and Acute Coronary Syndromes. Cardiol. Clin. 2018, 36, 1-12. [CrossRef]

5. Barón-Esquivias, G.; Santana-Cabeza, J.J.; Haro, R.; Núñez, A.; Pérez, E.; Martínez, Á.; Martínez-Rubio, A. Transtelephonic electrocardiography for managing out-of-hospital chest pain emergencies. J. Electrocardiol. 2011, 44, 755-760. [CrossRef]

6. Papai, G.; Racz, I.; Czuriga, D.; Szabo, G.; Edes, I.F.; Edes, I. Transtelephonic electrocardiography in the management of patients with acute coronary syndrome. J. Electrocardiol. 2014, 47, 294-299. [CrossRef]

7. Thiele, H.; Ohman, E.M.; Desch, S.; Eitel, I.; De Waha, S. Management of cardiogenic shock. Eur. Heart J. 2015, 36, 1223-1230. [CrossRef]

8. Vahdatpour, C.; Collins, D.; Goldberg, S. Cardiogenic Shock. J. Am. Heart Assoc. 2019, 8, e011991. [CrossRef]

9. Harjola, V.P.; Lassus, J.; Sionis, A.; Køber, L.; Tarvasmäki, T.; Spinar, J.; Parissis, J.; Banaszewski, M.; Silva-Cardoso, J.; Carubelli, V.; et al. Clinical picture and risk prediction of short-term mortality in cardiogenic shock. Eur. J. Heart Fail. 2015, 17, 501-509. [CrossRef]

10. Ponikowski, P.; Voors, A.A.; Anker, S.D.; Bueno, H.; Cleland, J.G.F.; Coats, A.J.S.; Falk, V.; González-Juanatey, J.R.; Harjola, V.P.; Jankowska, E.A.; et al. 2016 ESC Guidelines for the diagnosis and treatment of acute and chronic heart failure: The Task Force for 
the diagnosis and treatment of acute and chronic heart failure of the European Society of Cardiology (ESC)Developed with the special contribution of the Heart Failure Association (HFA) of the ESC. Eur. Heart J. 2016, 37, 2129-2200. [CrossRef]

11. Killip, T., III; Kimball, J.T. Treatment of myocardial infarction in a coronary care unit. A two year experience with 250 patients. Am. J. Cardiol. 1967, 20, 457-464. [CrossRef]

12. Thiele, H.; Zeymer, U.; Neumann, F.J.; Ferenc, M.; Olbrich, H.G.; Hausleiter, J.; Richardt, G.; Hennersdorf, M.; Empen, K.; Fuernau, G.; et al. Intraaortic balloon support for myocardial infarction with cardiogenic shock. N. Engl. J. Med. 2012, 367, 1287-1296. [CrossRef] [PubMed]

13. Thiele, H.; Zeymer, U.; Neumann, F.J.; Ferenc, M.; Olbrich, H.G.; Hausleiter, J.; de Waha, A.; Richardt, G.; Hennersdorf, M.; Empen, K.; et al. Intra-aortic balloon counterpulsation in acute myocardial infarction complicated by cardiogenic shock (IABP-SHOCK II): Final 12 month results of a randomised, open-label trial. Lancet 2013, 382, 1638-1645. [CrossRef]

14. Neumann, F.J.; Sousa-Uva, M.; Ahlsson, A.; Alfonso, F.; Banning, A.P.; Benedetto, U.; Byrne, R.A.; Collet, J.P.; Falk, V.; Head, S.J.; et al. 2018 ESC/EACTS Guidelines on myocardial revascularization. Eur. Heart J. 2019, 40, 87-165. [CrossRef]

15. Koszegi, Z.; Balkay, L.; Galuska, L.; Varga, J.; Hegedus, I.; Fulop, T.; Balogh, E.; Jenei, C.; Szabo, G.; Kolozsvari, R.; et al. Holistic polar map for integrated evaluation of cardiac imaging results. Comput. Med. Imaging Graph. 2007, 31, 577-586. [CrossRef]

16. Koszegi, Z.; Maes, A.; Piessens, J.; Van de Werf, F.; Mortelmans, L. Segmental comparison between coronary angiography and positron emission tomography reveals low predictive value of epicardial flow for viability. Eur. Heart J. 1998, 19, 959-967. [CrossRef]

17. Vogelzang, M.; Vlaar, P.J.; Svilaas, T.; Amo, D.; Nijsten, M.W.; Zijlstra, F. Computer-assisted myocardial blush quantification after percutaneous coronary angioplasty for acute myocardial infarction: A substudy from the TAPAS trial. Eur. Heart J. 2009, 30, 594-599. [CrossRef]

18. Porto, I.; Hamilton-Craig, C.; De Maria, G.L.; Vergallo, R.; Cautilli, G.; Galiuto, L.; Burzotta, F.; Leone, A.M.; Niccoli, G.; Natale, L.; et al. Quantitative Blush Evaluator accurately quantifies microvascular dysfunction in patients with ST-elevation myocardial infarction: Comparison with cardiovascular magnetic resonance. Am. Heart. J. 2011, 162, 372-381.e372. [CrossRef]

19. Papai, G.; Csato, G.; Racz, I.; Szabo, G.; Barany, T.; Racz, A.; Szokol, M.; Sarman, B.; Edes, I.F.; Czuriga, D.; et al. The transtelephonic electrocardiogram-based triage is an independent predictor of decreased hospital mortality in patients with ST-segment elevation myocardial infarction treated with primary percutaneous coronary intervention. J. Telemed. Telecare 2020, 26, 216-222. [CrossRef]

20. Brunetti, N.D.; Di Pietro, G.; Aquilino, A.; Bruno, A.I.; Dellegrottaglie, G.; Di Giuseppe, G.; Lopriore, C.; De Gennaro, L.; Lanzone, S.; Caldarola, P.; et al. Pre-hospital electrocardiogram triage with tele-cardiology support is associated with shorter time-to-balloon and higher rates of timely reperfusion even in rural areas: Data from the Bari-Barletta/Andria/Trani public emergency medical service 118 registry on primary angioplasty in ST-elevation myocardial infarction. Eur. Heart J. Acute Cardiovasc. Care 2014, 3, 204-213. [CrossRef]

21. Chan, A.W.; Kornder, J.; Elliott, H.; Brown, R.I.; Dorval, J.F.; Charania, J.; Zhang, R.; Ding, L.; Lalani, A.; Kuritzky, R.A.; et al. Improved survival associated with pre-hospital triage strategy in a large regional ST-segment elevation myocardial infarction program. JACC Cardiovasc. Interv. 2012, 5, 1239-1246. [CrossRef] [PubMed]

22. Brunetti, N.D.; Bisceglia, L.; Dellegrottaglie, G.; Bruno, A.I.; Di Pietro, G.; De Gennaro, L.; Di Biase, M. Lower mortality with pre-hospital electrocardiogram triage by telemedicine support in high risk acute myocardial infarction treated with primary angioplasty: Preliminary data from the Bari-BAT public Emergency Medical Service 118 registry. Int. J. Cardiol. 2015, 185, 224-228. [CrossRef] [PubMed]

23. Brunetti, N.D.; De Gennaro, L.; Correale, M.; Santoro, F.; Caldarola, P.; Gaglione, A.; Di Biase, M. Pre-hospital electrocardiogram triage with telemedicine near halves time to treatment in STEMI: A meta-analysis and meta-regression analysis of non-randomized studies. Int. J. Cardiol. 2017, 232, 5-11. [CrossRef] [PubMed]

24. de Waure, C.; Cadeddu, C.; Gualano, M.R.; Ricciardi, W. Telemedicine for the reduction of myocardial infarction mortality: A systematic review and a meta-analysis of published studies. Telemed. J. E Health 2012, 18, 323-328. [CrossRef]

25. Sanchez-Ross, M.; Oghlakian, G.; Maher, J.; Patel, B.; Mazza, V.; Hom, D.; Dhruva, V.; Langley, D.; Palmaro, J.; Ahmed, S.; et al. The STAT-MI (ST-Segment Analysis Using Wireless Technology in Acute Myocardial Infarction) trial improves outcomes. JACC Cardiovasc. Interv. 2011, 4, 222-227. [CrossRef]

26. Nieminen, M.S.; Buerke, M.; Cohen-Solál, A.; Costa, S.; Édes, I.; Erlikh, A.; Franco, F.; Gibson, C.; Gorjup, V.; Guarracino, F.; et al. The role of levosimendan in acute heart failure complicating acute coronary syndrome: A review and expert consensus opinion. Int. J. Cardiol. 2016, 218, 150-157. [CrossRef]

27. Lim, H.S. Cardiogenic Shock: Failure of Oxygen Delivery and Oxygen Utilization. Clin. Cardiol. 2016, 39, 477-483. [CrossRef]

28. Jeger, R.V.; Radovanovic, D.; Hunziker, P.R.; Pfisterer, M.E.; Stauffer, J.C.; Erne, P.; Urban, P. Ten-year trends in the incidence and treatment of cardiogenic shock. Ann. Intern. Med. 2008, 149, 618-626. [CrossRef]

29. Kochar, A.; Al-Khalidi, H.R.; Hansen, S.M.; Shavadia, J.S.; Roettig, M.L.; Fordyce, C.B.; Doerfler, S.; Gersh, B.J.; Henry, T.D.; Berger, P.B.; et al. Delays in Primary Percutaneous Coronary Intervention in ST-Segment Elevation Myocardial Infarction Patients Presenting With Cardiogenic Shock. JACC Cardiovasc. Interv. 2018, 11, 1824-1833. [CrossRef]

30. Scholz, K.H.; Maier, S.K.G.; Maier, L.S.; Lengenfelder, B.; Jacobshagen, C.; Jung, J.; Fleischmann, C.; Werner, G.S.; Olbrich, H.G.; Ott, R.; et al. Impact of treatment delay on mortality in ST-segment elevation myocardial infarction (STEMI) patients presenting with and without haemodynamic instability: Results from the German prospective, multicentre FITT-STEMI trial. Eur. Heart J. 2018, 39, 1065-1074. [CrossRef] 
31. Hochman, J.S.; Sleeper, L.A.; Webb, J.G.; Dzavik, V.; Buller, C.E.; Aylward, P.; Col, J.; White, H.D. Early revascularization and long-term survival in cardiogenic shock complicating acute myocardial infarction. JAMA 2006, 295, 2511-2515. [CrossRef] [PubMed]

32. Thiele, H.; Ohman, E.M.; de Waha-Thiele, S.; Zeymer, U.; Desch, S. Management of cardiogenic shock complicating myocardial infarction: An update 2019. Eur. Heart J. 2019, 40, 2671-2683. [CrossRef] [PubMed] 